\title{
Does testicular anti-Müllerian hormone extend life?
}

\author{
Ian S. McLennan $\mathbb{1}^{1} \cdot$ Yih Harng Chong ${ }^{1,2}$
}

Received: 17 October 2016 / Accepted: 25 October 2016 / Published online: 7 November 2016

(C) Springer Science+Business Media New York 2016

Men tend to have shorter lives than women, with the gonads appearing to contributing to this. Gonadal hormones decline from midlife in men, but few elderly men become totally devoid of testicular hormones, the male equivalent of menopause [1]. The systemic metabolic demands created by gonadal hormones are therefore more extended in men than women. When the testicular influence is lessened by castration at a young age, the resulting eunuchs typically outlive intact men by over a decade [2]. This supports the theory that the testes facilitate reproductive success, at the expensive of longevity.

Paradoxically, Qayyum and Akbar report in this edition of Endocrine that circulating anti-Müllerian hormone (AMH) in men negatively associates with all cause mortality [3]. Men with higher levels of a gonadal hormone may be outliving men with lower levels of the hormone. This is a novel observation, which opens new lines of investigation.

Testosterone is synonymous to the testes for many people, with few being aware that the testes release multiple protein hormones, including $\mathrm{AMH}$, inhibin $\mathrm{B}$ and insulin like-3 (INSL3). The levels of these testicular hormones are largely independent in young men, as is the time course of their decline during ageing [1]. Hence, the levels of each testicular hormone are determined by a unique set of regulatory determinants, rather than by a single pan-testicular mechanism. This points to each of the four hormones

Ian S. McLennan

ian.mclennan@otago.ac.nz

1 Department of Anatomy, School of Biomedical Sciences, University of Otago, Dunedin, New Zealand

2 Department of Medicine, Dunedin School of Medicine, University of Otago, Dunedin, New Zealand having distinct functions. Qayyum and Akbar's study [3] raises the possibility that AMH may partially counterbalances the life-shortening effects of other aspects of the testes.

Young men and women initially have similar levels of circulating levels of AMH, but young women lose their AMH in parallel with the decrease in their ovarian reserve. This adds to the paradox: AMH is the first of the ovarian hormones to decline, yet it associates with longevity in men, despite longevity having a female bias. AMH has two distinct faces (reviewed in [4]), and correlative studies do not establish which (if either) of the faces is important. At the start of life, $\mathrm{AMH}$ is a male-specific hormone, whose hallmark function is to induce the regression of the Müllerian duct, the uterine anlagen. The immature testes, however, continues to secrete very high levels of AMH into blood until puberty. Consequently, male embryos and boys develop with levels of AMH that do not occur during normal adult physiology or in developing females. The target tissues of AMH during development appear to be broad, but direct evidence of AMH actions is limited to the lungs and the brain. The correlates reported by Qayyum and Akbar [3] may be a reflection of this developmental mechanism, in which case they would be the product of male-specific biology. Men and women have low levels of circulating AMH compared to boys, but the adult levels are capable of signaling in some bioassays. If circulating AMH in adults maintains aspects of their homeostasis, as some animal studies suggest [5], then Qayyum and Akbar's [3] results are possibly important to both sexes. At the very least, this highlights the potential risks associated with administering the sex steroid hormones alone, without understanding whether the other gonadal hormones modulate or counterbalance the actions of the gonadal steroids. 
Correlation does not prove causality, and a single correlative study in an area within a small literature is far from definitive. The value of Qayyum and Akbar's study [3] is that it highlights what is unknown, and why various questions need to be addressed. The testis is a complex organ with two endocrine cells, each of which can independently release multiple hormones. Moving forward, we argue that the study of the testes should become synonymous with the measurement of all the testicular hormones. Testosterone is central to making an individual a man, but the balance of the multiple testicular hormones may define where in the spectrum of male phenotypes a man sit, both physiologically and clinically.

Funding The authors research is funded by The Health Research Council, New Zealand (14/441).

\section{Compliance with Ethical Standards}

Conflict of Interest The authors declare that they have no competing interests.

\section{References}

1. Y.H. Chong, M.P. Pankhurst, I.S. McLennan, The testicular hormones $\mathrm{AMH}$, inhb, insl3 and testosterone can be independently deficient in older men. J. Gerontol. A. (2016). doi:10.1093/gerona/ glw143

2. J.D. Wilson, C. Roehrborn, Long-term consequences of castration in men: Lessons from the skoptzy and the eunuchs of the chinese and ottoman courts. J. Clin. Endocrinol. Metab. 84, 4324-4331 (1999)

3. R. Qayyum, S. Akbar, Serum anti-mullerian hormone and all-cause mortality in men. Endocrine 54, 225-231 (2016)

4. I.S. McLennan, M.W. Pankhurst, Anti-mullerian hormone is a gonadal cytokine with two circulating forms and cryptic actions. J. Endocrinol. 226, R45-R57 (2015)

5. N. Lebeurrier, S. Launay, R. Macrez, E. Maubert, H. Legros, A. Leclerc, S.P. Jamin, J.Y. Picard, S. Marret, V. Laudenbach, P. Berger, P. Sonderegger, C. Ali, N. di Clemente, D. Vivien, Antimullerian-hormone-dependent regulation of the brain serineprotease inhibitor neuroserpin. J. Cell. Sci. 121, 3357-3365 (2008) 